\title{
Development of A Small-Scale Office Grid Computing System For Electromagnetic Optimization Designs
}

\author{
Ming-Iu Lai ${ }^{(1)}$ and Shyh-Kang Jeng ${ }^{(2)}$ \\ (1) Graduate Institute of Communication Engineering, \\ National Taiwan University, Taiwan, R.O.C. \\ milai1102@yahoo.com.tw \\ (2) Graduate Institute of Communication Engineering and \\ Department of Electronic Engineering, \\ National Taiwan University, Taiwan, R.O.C. \\ skjeng@ew.ee.ntu.edu.tw
}

A Small-Scale Office Grid Computing System for electromagnetic optimization designs is presented in this paper. This system is to collect the unused computational power together and to utilize up to $90 \%$ of computer resources in the office. The scale of the proposed system is set to connect up to 100 computational resources which is typical in an office environment. TCP/IP and winsock programming techniques are used to construct the networking between the job control manager and computational workers. Based on the proposed computing system, a multi-band printed monopole antenna design is demonstrated in this paper. The numerical electromagnetic technique used to solve the example is the FDTD method and the optimization is based on the genetic algorithms.

\section{Introduction}

In practical electromagnetic design, it is common to simulate the electrical performance using various numerical electromagnetic techniques such as the Finite Element Method (FEM) and the Finite-Difference Time-Domain (FDTD) method. In order to obtain a better design, it is usual to apply various optimization techniques to search for the potential solution. However, with a considerable number of design parameters to be optimized, it's frequently necessary to solve EM problem hundreds of times until the solution converges [1]. Because numerical EM solutions are often computationally intensive, the use of numerical solutions during the optimization process on a single computer is almost impossible. One way to perform these intensive tasks is to build a supercomputer. Nevertheless, this approach is very expensive. A much cheaper alternative is to use distributed computing.

In a typical office and laboratory environment, more and more computers appear, but the vast majority of the machines are used mostly for word processing, web browsing, files downloading, etc. The CPU time is often wasted doing nothing. This implies that more than $90 \%$ of computing resources are unused. Distributed computing technique is to utilize the spare computing power of every available computer, and to work by splitting up the larger task into smaller chunks, which can be performed at the same time independently of each other. In this way, the 
computing power of thousands of computers can be salvaged and combined together to solve a single problem. Distributed computing roughly falls into two categories, Cluster and Grid Computing. Cluster Computing, or so called PC Clusters, is a local computing system comprising a set of independent computers usually residing in a single room and a dedicated network interconnecting tightly the computers. Grid Computing focuses on ensembles of geographically distributed heterogeneous resources used as a platform for high performance computing. The computers need not be in the same room. Clusters and Grids [2] have been successfully applied in life sciences, aerospace, CAD/CAM, military applications, and so on. To solve large electromagnetic optimization problems, we need a computing system enabling us to easily access the computational resources in an office environment and is seamlessly compatible with various in-house numerical electromagnetic programs and optimization schemes.

In this paper, the concept is to develop a Small-Scale Office Grid Computing System for electromagnetic optimization designs using $\mathrm{C}++$ Object-Oriented Programming, TCP/IP and winsock programming techniques. Differing from the well-known distributed computing systems, the scale of the proposed system is set to connect up to 100 computational resources which is typical in an office environment. As an example, a multi-band printed monopole antenna design is demonstrated. The Genetic Algorithms (GAs) and the FDTD method are used for the design.

\section{Small-Scale Office Grid Computing System}

Figure 1 illustrates a basic networking architecture in offices or laboratories. The two main entities in the computing system are one manager and many computational workers. The manager generates job packages, which are passed onto computational workers. From the view of optimization, a job is an independent electromagnetic problem, and the job package defines which numerical program to be used to solve this EM problem, which files to be passed to a worker and returned to manager side, and so on. The workers will perform the task in the job package, and when it is finished, the calculated results will be passed back to the manager. The network topology is a star one. Because the amount of data exchange between the manager and a worker is just several tens of kilobytes, the communication time does not dominate the computing time in this system when the number of workers increases. Figure 2 displays the software architecture, where each box is implemented by a class. Block A consisting of two classes, CommunicationManager and WorkerClass, is a client-server communication program constructed by TCP/IP and winsock programming techniques [3]. The login information of a worker contains the name, IP address, memory space, computing capability, etc. According to the information, it is easy for the manager to know the computing limitation of each worker. The class JobManager manages the submitted job packages. Block B is composed of many classes designed according to different optimization schemes and various 
applications. The job packages are generated by these classes in this block and then submitted to the class JobManager for computing.

When receiving a job package from the manager side, the worker will create a thread with priority lower than normal to execute the job. The use of the lower priority is for the sake of making sure that the job does not interrupt local user's works. While the job is running, the class WorkerClass will periodically respond to the class CommunicationManager the series number of the governing job package. By means of this simple message, the manager is capable of identifying the job status. The job-synchronization problem depends on the optimization algorithm used, and is not discussed here for the limitation of spaces.

\section{Design Example}

Based on the proposed computing system, a multi-band printed monopole antenna design is demonstrated here. Printed monopole antennas are easy to be integrated on the printed circuit board (PCB), offering many features such as the small size and the low fabrication cost [4]. To satisfy the IEEE $802.11 \mathrm{a} / \mathrm{b} / \mathrm{g}$ standards, multi-band antennas are highly desired. The proposed system associated with FDTD and GA is to automatically design a multi-band monopole antenna with reflected coefficient lower than $-10 \mathrm{~dB}$ within $2.0-2.5$ and $5.0-6.0 \mathrm{GHz}$. The configuration of the antenna is shown in Fig. 3(a), and the design parameters are $\operatorname{len}_{0}, \operatorname{len}_{1}, \operatorname{len}_{2}, \operatorname{len}_{3}, \operatorname{len}_{4}, w_{0}, w_{1}, w_{2}, w_{3}, w_{4}, w_{5}, s_{1}, s_{2}, s_{3}$, and $s_{4}$. The antenna is designed on an FR4 substrate with thickness $h=0.8 \mathrm{~mm}$ and relative dielectric constant $\varepsilon_{r}=4.2$. The FDTD solution is solved in a cubic region of dimension $\left(N_{x}, N_{y}, N_{z}\right)=(151,151,34)$ grid points. The grids chosen are $\Delta x=\Delta y=\Delta z=0.25 \mathrm{~mm}$ and the total number of time steps is 3000 . One FDTD solution takes about 40 minutes at the best computational worker (with a Pentium $4 \mathrm{CPU}$ ) and about 2 hours at the worst one (with a Pentium $3 \mathrm{CPU}$ ). After the evolution of 20 generations, the simulated and measured reflected coefficients of the best solution are shown in Fig 3(b). The number of population for each generation is 20 , and the evaluation count is about 280 . The number of computational workers is between 5 and 11 , and the computing time is about 38hours.

\section{Conclusions}

Based on $\mathrm{C}++, \mathrm{TCP} / \mathrm{IP}$, and winsock programming techniques, a Small-Scale Office Grid Computing System for electromagnetic optimization designs has been proposed. This system can collect the unused computational power together and utilize up to $90 \%$ of computer resources in the office. Based on the proposed computing system, a multi-band monopole antenna design has been demonstrated in this paper. This example reveals that this system is efficient. The extension of this system to deal with general electromagnetic optimization is also under development. 


\section{References:}

[1] Yahya Ruhmat-Samii and Eric Michielssen, Electromagnetic Optimization by Genetic Algorithms, John Wiely \& Sons, Inc., 1999.

[2] Ian Foster, "The Grid: Computing without Bounds," Scientific American, Apr. 2003.

[3] Douglas E. Comer and David L. Stevens, Internetworking with TCP/IP Vol. III Client-Server Programming and Applications-Windows Sockets Version, Prentice Hall, 1997.

[4] Yen-Liang Kuo and Kin-Lu Wong, "Printed double-T monopole antenna for 2.4/5.2 GHz dual-band WLAN operations," IEEE Trans. Antenna Propagat., vol. 51 , pp. $2187-2192,2003$.

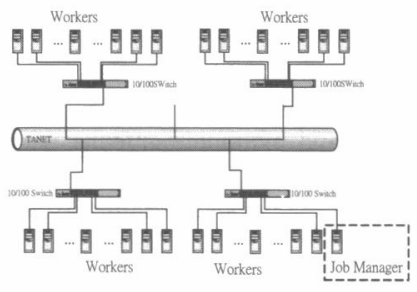

Fig. 1 Networking architecture of the proposed system.

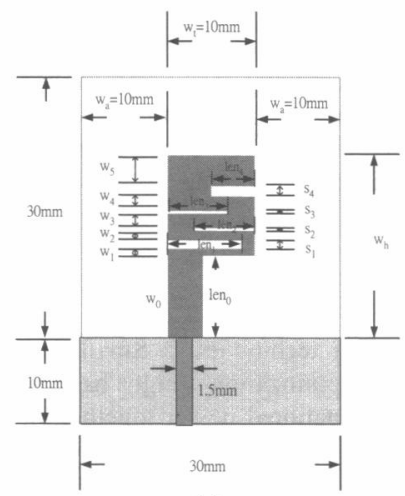

(a)

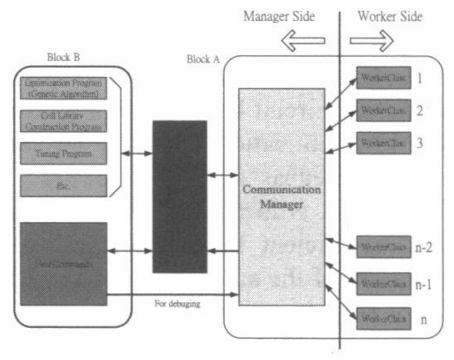

Fig. 2 Software architecture of the proposed system.

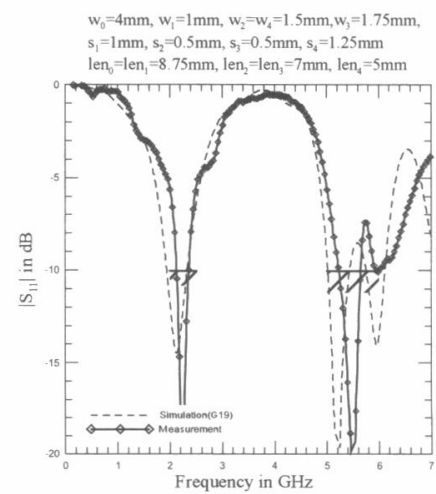

(b)

Fig. 3 A Multi-band printed monopole antenna. (a) Configuration. (b) Simulated and measured reflected coefficients of the best solution after the evolution of 20 generations. 